\title{
New Classification for Heart Failure with Mildly Reduced Ejection Fraction Greater clarity or more confusion?
}

\author{
Sunil Nadar
}

\begin{abstract}
ABSTRAC T: The latest European Society of Cardiology (ESC) guidelines for the diagnosis and management of heart failure include a new patient group for those with heart failure with mildly reduced ejection fraction (HFmrEF). By defining this group of patients as a separate entity, the ESC hope to encourage more research focusing on patients with HFmrEF. Previously, patients with this condition were caught between two classifications-heart failure with reduced ejection fraction and heart failure with preserved ejection fraction. Hopefully, the inclusion of new terminology will not increase confusion, but rather aid our understanding of heart failure, a complex clinical syndrome.
\end{abstract}

Keywords: Heart Failure; Ventricular Ejection Fraction; Guidelines as Topic; Nomenclature.

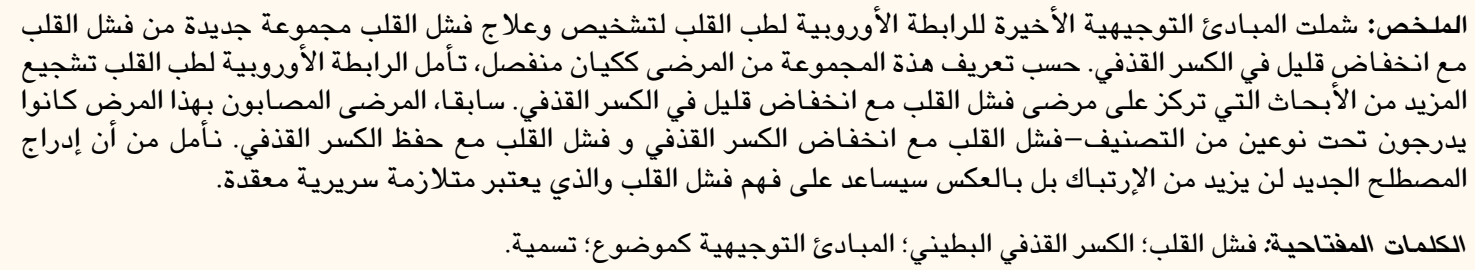

$\mathrm{J}$

UST AS GENERAL PHYSICIANS HAVE BECOME accustomed to the concept of heart failure with preserved ejection fraction (HFpEF), the latest 2016 guidelines for the diagnosis and treatment of heart failure from the European Society of Cardiology (ESC) have introduced a new group of patients: those with heart failure with mildly reduced ejection fraction (HFmrEF). ${ }^{1}$ The ESC has explained that this new category was needed as a means to fill the gap that existed in the knowledge and understanding of heart failure as well as stimulating research on this group of patients who have not traditionally been included in studies on heart failure with reduced ejection fraction (HFrEF) or HFpEF. ${ }^{1}$

In the 2016 ESC guidelines, the diagnostic criteria for HFmrEF includes signs and symptoms of heart failure, an ejection fraction (EF) of 40-49\%, elevated levels of natriuretic peptides and either relevant structural heart disease (i.e. left ventricular hypertrophy and/or left atrial enlargement) or diastolic dysfunction observed during echocardiography. ${ }^{1}$ Interestingly, the last two criteria are also used to diagnose HFpEF; this new category could therefore be considered to constitute either pre-HFrEF or progressive HFpEF, assuming that HFpEF is a forerunner of HFrEF., This is supported by the fact that the ventricles of patients with HFpEF are not entirely normal and have certain contractile abnormalities. ${ }^{4,5}$

\section{Previous Changes in Classifications}

In order to ascertain whether the new classification of HFmrEF is beneficial, it is useful to reflect on the introduction of the term 'HFpEF'. Prior to the formal identification of this group of patients as a distinct entity, clinicians were aware that some patients, mainly elderly hypertensive females, presented with clinical signs of heart failure but displayed normal left ventricular function on echocardiography. ${ }^{6}$ Various aetiologies were suggested and these patients were initially labelled as having diastolic heart failure, as it was believed that these hypertensive patients had stiff ventricles that did not relax. ${ }^{6}$ In the absence of any 
clinical evidence, clinicians often treated this group of patients in the same way as those with HFrEF, with varying results. ${ }^{7}$ It was Gandhi et al. who first reported 38 patients presenting with hypertensive acute pulmonary oedema and preserved left ventricular function, both during the acute phase and after successful treatment of their acute presentation, thus demonstrating that these patients constituted a distinct entity. ${ }^{8}$ This patient group was subsequently given many labels, such as 'diastolic heart failure' and 'heart failure with normal EF.' Eventually, the American Heart Association and the ESC formally accepted the term 'HFpEF' ${ }^{\prime 0,11}$

The formal recognition of $\mathrm{HFpEF}$ as a distinct entity has led to more focused research on this group of patients. It is now known that these patients are a heterogeneous group not limited to elderly hypertensive females and whose ventricles have normal systolic function with varying degrees of stiffness and diastolic abnormalities. ${ }^{12}$ In addition, evidence now shows that many of the treatment modalities for treating HFrEF are ineffective for patients with HFpEF. ${ }^{12}$ These findings have changed the management of and approach to dealing with patients with HFpEF, although there are admittedly still many unanswered questions. ${ }^{13}$ In a similar manner, despite the possible initial reluctance to accept another category of heart failure patients, the decision by the ESC to define HFmrEF may help to increase understanding of this disease and improve its treatment, particularly for patients who fall in between the two previous categories.

\section{Current Approaches to Diagnosis and Management}

Clinicians familiar with the management of heart failure patients will know that individuals with HFmrEF are often difficult to treat. In a way, they are somewhat similar to HFpEF patients whereby the symptoms of heart failure appear disproportional to the level of reduced EF; moreover, these patients do not often respond as well to diuretics, angiotensinconverting enzyme inhibitors or $\beta$-blockers. ${ }^{14}$ Similar to the treatment that HFpEF patients received prior to breakthroughs in the understanding of the disease, HFmrEF patients are still treated with similar management plans to those of HFrEF patients. ${ }^{15}$ Although there is often no evidence to back up these management plans, clinicians tend to rely on their clinical experience. Perhaps time will tell whether these patients are more likely to respond to conventional heart failure treatments or whether there is a need for more specific treatment modalities. Currently, the ESC guidelines have combined the management of patients with HFmrEF with that of HFpEF patients.

A major problem in the management of HFpEF, and now HFmrEF as well, is establishing the diagnosis. ${ }^{16}$ There is still some reluctance to label a patient with preserved left ventricular function as having heart failure; as such, HFpEF and HFmrEF patients are often underdiagnosed. ${ }^{16}$ These patients sometimes undergo multiple tests to rule out respiratory and ischaemic causes of breathlessness before ultimately being diagnosed with heart failure. Moreover, HFpEF and HFmrEF patient groups are heterogeneous and often have multiple comorbidities that can confuse the diagnosis. ${ }^{12}$ There is a danger of both the overand underdiagnosis of heart failure. Epidemiological studies have shown that the incidence of HFpEF appears to be on the rise. ${ }^{17,18}$ This could potentially be explained by the developing awareness of HFpEF on the part of clinicians and their increased willingness to make this diagnosis now that it has been officially recognised.

\section{Opportunities for Further Research}

With the previously undefined group of HFmrEF patients now having been legitimised with an official name, albeit one difficult to articulate properly, it can be hoped that this will spur research focusing specifically on this patient group. ${ }^{19}$ Currently, it is unknown whether these patients have a milder version of HFrEF, if HFmrEF indicates those with HFpEF whose EF is now deteriorating or indeed if it is simply a part of the heart failure continuum. ${ }^{19}$ Along those lines, it is not clear whether aggressive treatment of HFpEF would prevent patients from progressing to HFmrEF or whether aggressive management of HFmrEF would prevent patients from deteriorating to HFrEF. More research is therefore justified. This change in nomenclature gives rise to many questions that clinicians have perhaps always asked themselves, but never had the chance to openly query; these can hopefully now be answered, perhaps via large-scale clinical trials.

\section{Alternative Classifications of Heart Failure}

It must be noted that the use of EF as a method of classifying heart failure is not universally agreed upon. De Keulenaer et al. argue that HFpEF and HFrEF are overlapping phenotypes within the spectrum of heart failure and that the use of EF to classify heart failure is 
not accurate. ${ }^{3}$ They suggest that this classification came about primarily because early heart failure drug trials included patients with low EF, who were considered a high-risk group, in order to produce statistically significant results. ${ }^{3}$ Subsequently, an emphasis on evidence-based medicine has automatically led to the use of cut-off values derived from the results of these trials. Another suggestion is that the disease be classified based on aetiology or physiological abnormalities, as well as the clinical presentation of acute or chronic heart failure symptoms, as this affects management choices. ${ }^{9}$ Furthermore, by using pathophysiology to classify heart failure, other researchers have proposed the use of biomarkers either alone or in combination with echocardiography in order to guide therapy and management choices, instead of relying entirely on EF measurements. ${ }^{20-22}$

\section{Conclusion}

Despite the announcement by the ESC of yet another category of heart failure patients with a tonguetwisting acronym, it is unlikely that the clinical management of this new HFmrEF patient group will change immediately. However, this new classification does define another subset of heart failure patients on whom additional and more focused research can now be conducted. Ideally, this will ensure increased understanding of this patient group in order to improve and effectively tailor management approaches.

\section{References}

1. Ponikowski P, Voors AA, Anker SD, Bueno H, Cleland JG, Coats AJ, et al. 2016 ESC guidelines for the diagnosis and treatment of acute and chronic heart failure: The task force for the diagnosis and treatment of acute and chronic heart failure of the European Society of Cardiology (ESC) - Developed with the special contribution of the Heart Failure Association (HFA) of the ESC. Eur Heart J 2016; 37:2129-200. doi: 10.1093/ eurheartj/ehw128.

2. Cahill JM, Ryan E, Travers B, Ryder M, Ledwidge $M$, McDonald K. Progression of preserved systolic function heart failure to systolic dysfunction: A natural history study. Int J Cardiol 2006; 106:95-102. doi: 10.1016/j.ijcard.2004.12.096.

3. De Keulenaer GW, Brutsaert DL. Systolic and diastolic heart failure are overlapping phenotypes within the heart failure spectrum. Circulation 2011; 123:1996-2004. doi: 10.1161/CIR CULATIONAHA.110.981431.

4. Wang J, Khoury DS, Yue Y, Torre-Amione G, Nagueh SF. Preserved left ventricular twist and circumferential deformation, but depressed longitudinal and radial deformation in patients with diastolic heart failure. Eur Heart J 2008; 29:1283-9. doi: 10.1093/eurheartj/ehn141.

5. Yu CM, Lin H, Yang H, Kong SL, Zhang Q, Lee SW. Progression of systolic abnormalities in patients with "isolated" diastolic heart failure and diastolic dysfunction. Circulation 2002; 105:1195-201. doi: 10.1161/hc1002.105185.
6. Vasan RS, Levy D. Defining diastolic heart failure: A call for standardized diagnostic criteria. Circulation 2000; 101:2118-21. doi: 10.1161/01.CIR.101.17.2118.

7. Dauterman KW, Massie BM, Gheorghiade M. Heart failure associated with preserved systolic function: A common and costly clinical entity. Am Heart J 1998; 135:S310-19. doi: 10.1016/S0002-8703(98)70258-3.

8. Gandhi SK, Powers JC, Nomeir AM, Fowle K, Kitzman DW, Rankin KM, et al. The pathogenesis of acute pulmonary edema associated with hypertension. N Engl J Med 2001; 344:17-22. doi: 10.1056/NEJM200101043440103.

9. Sanderson JE. HFNEF, HFpEF, HF-PEF, or DHF: What is in an acronym? JACC Heart Fail 2014; 2:93-4. doi: 10.1016/j.jchf. 2013.09.006.

10. Jessup M, Abraham WT, Casey DE, Feldman AM, Francis GS, Ganiats TG, et al. 2009 focused update: ACCF/AHA Guidelines for the Diagnosis and Management of Heart Failure in Adults - A report of the American College of Cardiology Foundation/American Heart Association Task Force on Practice Guidelines: Developed in collaboration with the International Society for Heart and Lung Transplantation. Circulation 2009; 119:1977-2016. doi: 10.1161/CIRCULATIO NAHA.109.192064.

11. McMurray JJ, Adamopoulos S, Anker SD, Auricchio A, Böhm M, Dickstein $\mathrm{K}$, et al. ESC guidelines for the diagnosis and treatment of acute and chronic heart failure 2012: The Task Force for the Diagnosis and Treatment of Acute and Chronic Heart Failure 2012 of the European Society of Cardiology Developed in collaboration with the Heart Failure Association (HFA) of the ESC. Eur J Heart Fail 2012; 14:803-69. doi: 10.1093/eurihf/hfs105.

12. Sharma K, Kass DA. Heart failure with preserved ejection fraction: Mechanisms, clinical features, and therapies. Circ Res 2014; 115:79-96. doi: 10.1161/CIRCRESAHA.115.302922.

13. Ferrari R, Böhm M, Cleland JG, Paulus WJ, Pieske B, Rapezzi C, et al. Heart failure with preserved ejection fraction: Uncertainties and dilemmas. Eur J Heart Fail 2015; 17:665-71. doi: $10.1002 /$ ejhf.304

14. Lam CS, Solomon S. The middle child in heart failure: Heart failure with mid-range ejection fraction (40-50\%). Eur J Heart Fail 2014; 16:1049-55. doi: 10.1002/ejhf.159.

15. Maggioni AP, Anker SD, Dahlström U, Filippatos G, Ponikowski P, Zannad F, et al. Are hospitalized or ambulatory patients with heart failure treated in accordance with European Society of Cardiology guidelines? Evidence from 12,440 patients of the ESC Heart Failure Long-Term Registry. Eur J Heart Fail 2013; 15:1173-84. doi: 10.1093/eurjhf/hft134.

16. Caruana L, Petrie MC, Davie AP, McMurray JJ. Do patients with suspected heart failure and preserved left ventricular systolic function suffer from "diastolic heart failure" or from misdiagnosis? A prospective descriptive study. BMJ 2000; 321:215-18. doi: 10.1136/bmj.321.7255.215.

17. Roger VL, Weston SA, Redfield MM, Hellermann-Homan JP, Killian J, Yawn BP, et al. Trends in heart failure incidence and survival in a community-based population. JAMA 2004; 292:344-50. doi: 10.1001/jama.292.3.344.

18. Owan TE, Hodge DO, Herges RM, Jacobsen SJ, Roger VL, Redfield MM. Trends in prevalence and outcome of heart failure with preserved ejection fraction. N Engl J Med 2006; 355:251-9. doi: 10.1056/NEJMoa052256.

19. Borlaug BA, Redfield MM. Diastolic and systolic heart failure are distinct phenotypes within the heart failure spectrum. Circulation 2011; 123:2006-13. doi: 10.1161/CIRCULATION AHA.110.954388. 
20. Troughton RW, Frampton CM, Brunner-La Rocca HP, Pfisterer M, Eurlings LW, Erntell $\mathrm{H}$, et al. Effect of B-type natriuretic peptide-guided treatment of chronic heart failure on total mortality and hospitalization: An individual patient meta-analysis. Eur Heart J 2014; 35:1559-67. doi: 10.1093/ eurheartj/ehu090.

21. Troughton R, Michael Felker G, Januzzi JL Jr. Natriuretic peptide-guided heart failure management. Eur Heart J 2014; 35:16-24. doi: 10.1093/eurheartj/eht463.
22. Simioniuc A, Carluccio E, Ghio S, Rossi A, Biagioli P, Reboldi G, et al. Echo and natriuretic peptide guided therapy improves outcome and reduces worsening renal function in systolic heart failure: An observational study of 1137 outpatients. Int J Cardiol 2016; 224:416-23. doi: 10.1016/j. ijcard.2016.09.034.

\section{ACCESS TO FULL-TEXT ARTICLES}

All articles published in SQUMJ can be found online in PubMed and the full text is freely available on PubMed Central and the SQUMJ content website. 